\title{
CSF3R T618I mutant chronic myelomonocytic leukemia (CMML) defines a proliferative CMML subtype enriched in ASXL1 mutations with adverse outcomes
}

\author{
Evandro D. Bezerra ${ }^{1}$, Terra L. Lasho ${ }^{1}$, Christy M. Finke ${ }^{1}$, Antoine N. Saliba', Michelle A. Elliott ${ }^{1}$, Animesh D. Pardanani ${ }^{1}$, \\ Naseema Gangat (1), Abhishek A. Mangaonkar', Rhett P. Ketterling ${ }^{2}$, Ayalew Tefferi ${ }^{1}$, Eric Solary $\mathbb{B}^{3,4}$ and \\ Mrinal M. Patnaik (iD ${ }^{1}$
}

Dear Editor,

Chronic myelomonocytic leukemia (CMML) is a clonal myeloid disorder characterized by peripheral blood (PB) monocytosis (absolute monocyte count (AMC) $\geq 1 \times 10^{9} / \mathrm{L}$ and $\geq 10 \%$ of the total white blood cell count (WBC)) and overlapping features of myelodysplastic syndromes and myeloproliferative neoplasms (MPN) ${ }^{1}$. Sustained PB monocytosis is a hallmark of CMML, with a mutational signature consisting of biallelic TET2, or TET2 and SRSF2 co-mutations, biasing hematopoiesis toward monocytosis ${ }^{2}$. Chronic neutrophilic leukemia $(\mathrm{CNL})$ is a rare MPN characterized by sustained mature neutrophilia $(\mathrm{WBC} \geq$ $25 \times 10^{9} / \mathrm{L}$, with segmented neutrophils and band forms $\geq 80 \%$ of the WBC), with an AMC $<1 \times 10^{9} / \mathrm{L}^{3}$. CNL commonly occurs due to oncogenic driver mutations involving the colony-stimulating factor receptor, CSF3R $(90-100 \%)^{4,5}$. Among CSF3R mutations described in CNL, the T6181 membrane proximal point mutation activating Janus-activated kinase (JAK)/signal transducer and activator of transcription factor signaling is the most common $(74 \%)^{4,6}$. These mutations potentially render CNL responsive to therapy with JAK inhibitors, such as ruxolitinib ${ }^{7}$. On the other hand, CSF $3 R$ mutations, especially CSF3R T618I, are very infrequent in CMML $(<5 \%)^{8,9}$. We carried out this study to assess the prevalence,

\footnotetext{
Correspondence: Mrinal M. Patnaik (patnaik.mrinal@mayo.edu)

${ }^{1}$ Division of Hematology, Mayo Clinic, Rochester, MN, USA

${ }^{2}$ Department of Laboratory Medicine and Pathology, Mayo Clinic, Rochester, MN, USA

Full list of author information is available at the end of the article
}

phenotypic features, and outcomes of patients with CMML who harbor the CSF3R T618I mutation.

Eight hundred and forty-six patients with CMML, defined per the World Health Organization (WHO) 2016 criteria, from Mayo Clinic, Minnesota and the Groupe Francais des Myelodysplasies were included in this study (August 1994 through July 2019). All patients underwent targeted next-generation sequencing for myeloid-relevant genes obtained at diagnosis or at first referral using previously described methods ${ }^{2,10}$. Categorical variables were compared by Fisher's exact or Pearson's chi square. Continuous variables were compared by Wilcoxon test. Overall survival (OS) was defined as the time from diagnosis to death. Acute myeloid leukemia-free survival (AML-FS) was defined as the time from diagnosis to transformation to AML or death. Patients who underwent hematopoietic cell transplantation prior to AML transformation were censored for AML-FS estimates. Both OS and AML-FS were estimated by the Kaplan-Meier method and compared by log-rank test.

We identified 6 (1\%) patients who met the 2016 WHO criteria for CMML and who harbored CSF3R mutations, all of whom had the CSF3R T618I missense alteration; 3 (50\%) were males, median age 63 years, with a median AMC of $6 \times 10^{9} / \mathrm{L}$ (range 2-23). Five (100\%) of five evaluable patients had bone marrow (BM) dysplasia, a finding not seen in CNL. As expected, all six CSF3R T618I mutant patients had a proliferative CMML phenotype, with marked neutrophilia in addition to the monocytosis. Four $(100 \%)$ of the 4 evaluable patients also had circulating immature myeloid cells (myelocytes and metamyelocytes). 


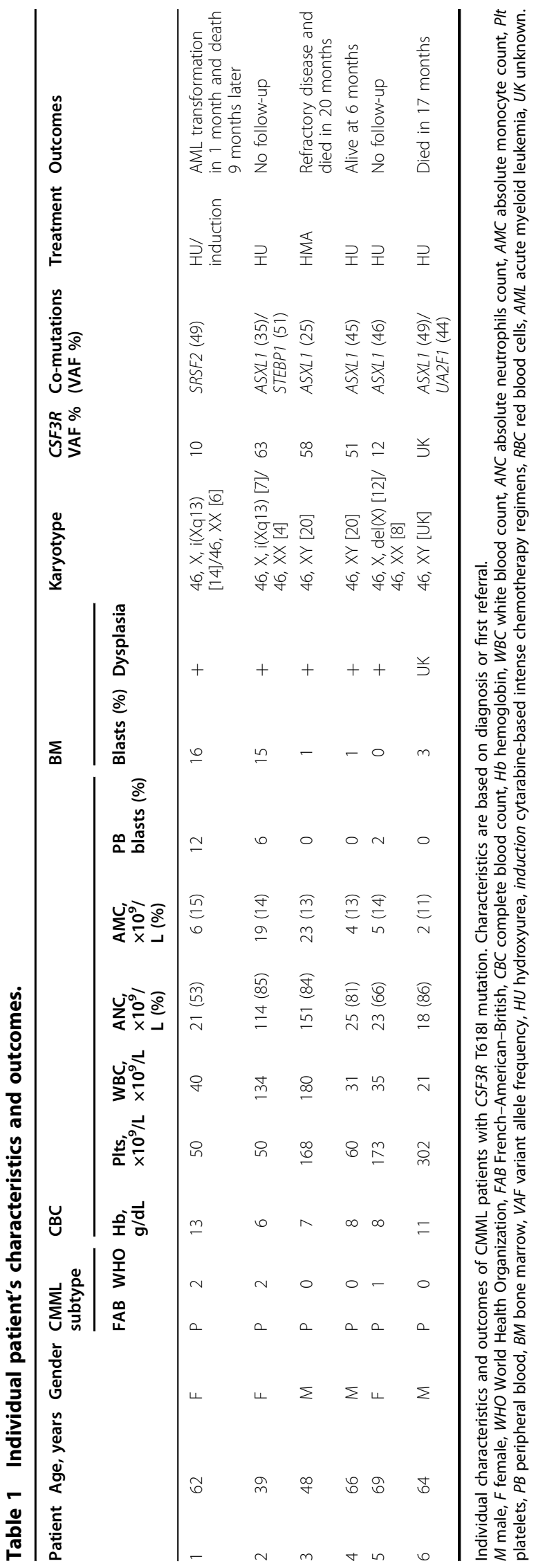

The CSF3R T618I variant allele frequency (VAF) burdens were $>40 \%$ in $3(60 \%)$ of 5 evaluable cases (range 10-63\%). While the CSF3R mutational VAF in three patients was in the heterozygous range, all three had adult-onset leukocytosis/monocytosis, making the likelihood that these variants were germline, quite low $^{11}$. We, however, acknowledge that we did not have germline tissue for further confirmation. Three patients had a normal karyotype, while the remaining three had $\mathrm{X}$ chromosome abnormalities (Table 1).

In comparison to CSF3R wild-type (WT) CMML patients, those with the CSF3R T618I mutation were younger (median 63 vs. 72 years; $p=0.0055$ ), had lower hemoglobin values ( $\mathrm{Hb}$, median 8 vs. $11 \mathrm{~g} / \mathrm{dl} ; p=0.0186)$, higher WBC (median 38 vs. $13 \times 10^{9} / \mathrm{L} ; p=0.0013$ ), higher percentage of neutrophils (median 83 vs. $52 \%$; $p=$ 0.001 ), higher AMC (median 6 vs. $2 \times 10^{9} / \mathrm{L} ; p=0.024$ ), lower percentage of monocytes (median 13 vs. $23 \%$; $p=$ 0.0069 ), higher percentage of PB blasts (median 1 vs. $0 \%$; $p=0.0472$ ), and were more likely to be classified as proliferative CMML (100 vs. $48 \%$; $p=0.012$ ). There were no TET2 mutations in the CSF3R T618I mutant CMML group, vs. $59 \%$ in the WT group ( $p=0.005$ ), while ASXL1 mutations were more frequent in the CSF3R T618I mutant group (83 vs. $41 \%$; $p=0.045$ ) in comparison to the WT group (Supplementary Table 1). While not statistically significant, 5 of the 6 (83\%) CSF3R T618I mutant CMML patients did not have concomitant SRSF2 mutations, with 4 of the $6(66 \%)$ not having any splicing mutation at all.

At last follow-up, 3 (50\%) and 400 (48\%) deaths and 1 $(17 \%)$ and $122(15 \%)$ leukemic transformations were documented in the CSF3R T618I mutant and WT CMML groups, respectively. The median OS was significantly shorter in CMML patients with the CSF3R T618I mutation (median 1.4 vs. 3.0 years; $p=0.049$ ), in comparison to the WT patients, with no difference in the AML-FS (1.4 vs. 3.2 years; $p=0.17$ ) (Fig. 1). None of our patients received ruxolitinib or underwent allogeneic stem cell transplantation. Five of the six patients were treated with hydroxyurea and one patient was treated with hypomethylating agent therapy, with no response.

Given the higher prevalence of $A S X L 1$ mutations in the CSF3R T618I mutated cohort, and the known detrimental prognostic impact of ASXL1 mutations in CMML, we performed a subgroup analysis comparing CSF3R T618I mutant CMML with ASXL1 mutant CMML patients (Supplementary Table 1$)^{10}$. In this comparison, once again, CSF3R mutant CMML patients were more likely to be younger in age $(p=0.009)$, have lower Hb levels $(p=$ $0.005)$, have higher WBC $(p=0.009)$, have lower AMC $(p=0.012)$, and were less likely to have TET2 mutations $(p=0.029)$. There was no OS difference between the two groups (Supplementary Fig. 1). 


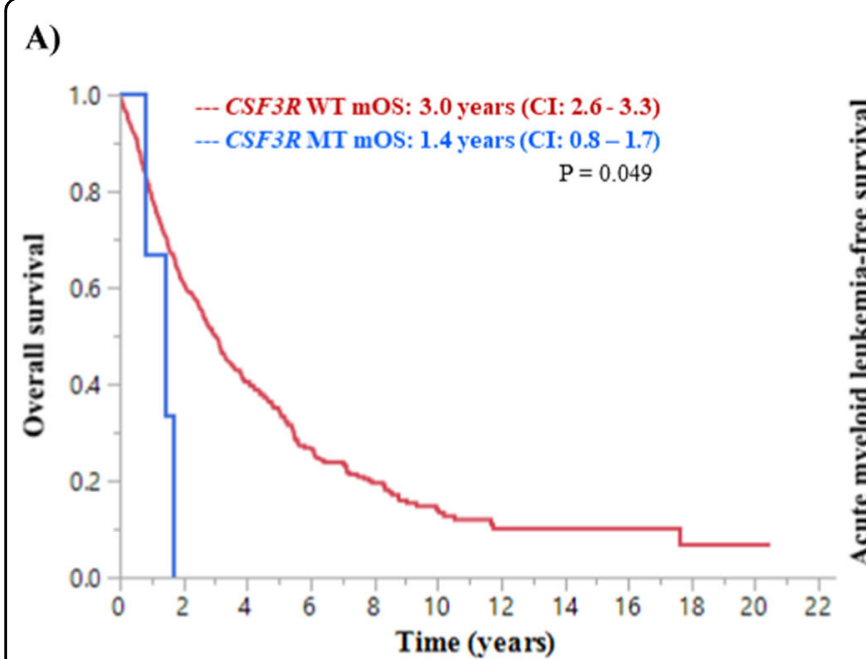

B)

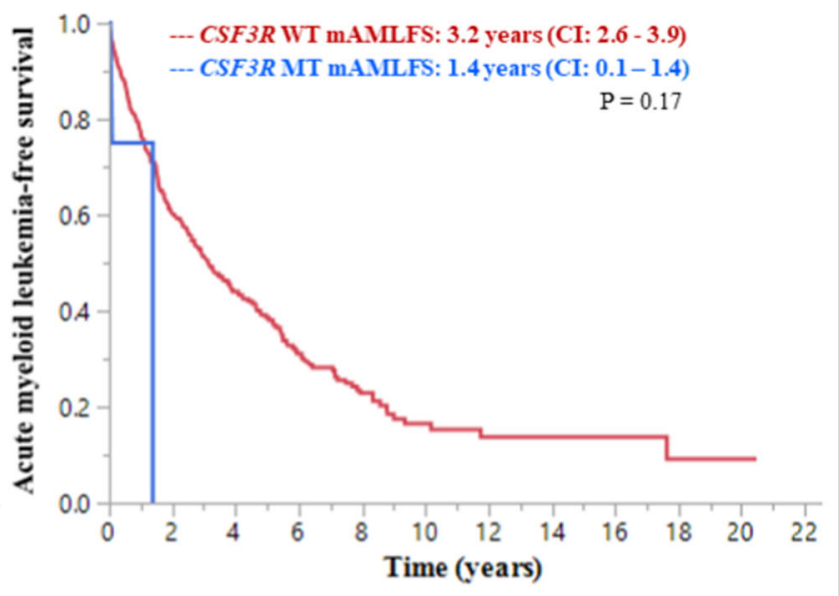

Fig. 1 Kaplan-Meier survival analysis of CMML patients stratified by the presence or absence of the CSF3RT618I mutation. A Inferior overall survival of CSF3RT618I mutant CMML in comparison to CSF3R wildtype patients. B Inferior acute myeloid leukemia-free survival of CSF3RT618I mutant CMML in comparison to CSF3R wildtype patients.

In summary, we present a cohort of patients who met 2016 WHO criteria for CMML and who harbored an oncogenic driver mutation in CSF3R T618I. While this mutation is fairly specific to CNL, infrequent occurrences in other myeloid neoplasms have been documented ${ }^{8,9}$. In our opinion, in the context of accurate histopathological analyses, the true frequency of these mutations in CMML is $<1 \%$. In addition, the CSF3R T618I mutation when present defines a unique proliferative CMML subtype characterized by pronounced leukocytosis/neutrophilia/monocytosis, with a unique molecular signature (ASXL1mt/TET2wt), with infrequent splicing mutations. The high frequency of $\mathrm{X}$ chromosome abnormalities remains to be defined. We have in the past demonstrated the negative prognostic impact of the $A S X L 1 \mathrm{mt} / T E T 2 \mathrm{wt}$ genotype in CMML, a finding once again validated by the poor OS of this group in the current study (median OS 1.4 years) ${ }^{2,12}$. In fact, the molecular landscape of CSF3R T618I mutant CMML is more akin to $B C R-A B L 1$-negative atypical chronic myeloid leukemia (CML), where ASXL1 mutations are frequent $(80 \%)$ and splicing mutations are less common $(30 \%)^{13}$. However, atypical CML is not associated with monocytosis, and given the fact that in CMML biallelic TET2 or TET2/SRSF2 comutations skew hematopoiesis toward monocytosis, the etiology of monocytosis in these patients remains to be elucidated. While the presence of BM dysplasia along with concomitant monocytosis/neutrophilia morphologically distinguishes CSF3R T618I mutant CMML from CNL, further work is needed to see whether CSF3R T618I mutant CMML is truly a CMML subtype, or if akin to PDGFRA/B and FGFR1 rearranged myeloid neoplasms, it deserves its own classification schema as a CSF3R T618I mutant myeloid neoplasm with monocytosis.

\section{Author details}

${ }^{1}$ Division of Hematology, Mayo Clinic, Rochester, MN, USA. ${ }^{2}$ Department of Laboratory Medicine and Pathology, Mayo Clinic, Rochester, MN, USA. ${ }^{3}$ Faculte de Medecine, Universite Paris-Saclay, 94270 Le Kremlin-Bicetre, France. ${ }^{4}$ Department of Hematology and INSERM U1287, Gustave Roussy Cancer Center, 94805 Villejuif, France

\section{Author contributions}

E.D.B. collected and interpreted the data, performed statistical analysis, and wrote the manuscript; T.L.L. interpreted the data and reviewed the manuscript; C.M.F. collected the data; A.N.S., M.A.E., A.D.P., N.G., A.A.M., R.P.K., A.T., and E.S reviewed the manuscript; and M.M.P. concepted the study, interpreted the data, and revised the manuscript.

\section{Conflict of interest}

The authors declare no competing interests.

\section{Publisher's note}

Springer Nature remains neutral with regard to jurisdictional claims in published maps and institutional affiliations.

Supplementary information The online version contains supplementary material available at https://doi.org/10.1038/s41408-021-00449-9.

Received: 28 November 2020 Revised: 12 January 2021 Accepted: 25 January 2021

Published online: 11 March 2021

\section{References}

1. Arber, D. A. et al. The 2016 revision to the World Health Organization classification of myeloid neoplasms and acute leukemia. Blood. 127, 2391-2405 (2016).

2. Coltro, G. et al. Clinical, molecular, and prognostic correlates of number, type, and functional localization of TET2 mutations in chronic myelomonocytic leukemia (CMML)-a study of 1084 patients. Leukemia 34, 1407-1421 (2020).

3. Szuber, N., Elliott, M. \& Tefferi, A. Chronic neutrophilic leukemia: 2020 update on diagnosis, molecular genetics, prognosis, and management. Am. J. Hematol. 95, 212-224 (2020).

4. Maxson, J. E. et al. Oncogenic CSF3R mutations in chronic neutrophilic leukemia and atypical CML. N. Engl. J. Med. 368, 1781-1790 (2013). 
5. Pardanani, A. et al. CSF3R T618l is a highly prevalent and specific mutation in chronic neutrophilic leukemia. Leukemia 27, 1870-1873 (2013).

6. Szuber, N. et al. CSF3R-mutated chronic neutrophilic leukemia: long-term outcome in 19 consecutive patients and risk model for survival. Blood Cancer J. 8, 21 (2018).

7. Dao, K. T. et al. Efficacy of ruxolitinib in patients with chronic neutrophilic leukemia and atypical chronic myeloid leukemia. J. Clin. Oncol. 38, 1006-1018 (2020).

8. Kosmider, $\mathrm{O}$. et al. Mutation of the colony-stimulating factor-3 receptor gene is a rare event with poor prognosis in chronic myelomonocytic leukemia. Leukemia 27, 1946-1949 (2013).

9. Ouyang, Y., Qiao, C., Chen, Y. \& Zhang, S. J. Clinical significance of CSF3R, SRSF2 and SETBP1 mutations in chronic neutrophilic leukemia and chronic myelomonocytic leukemia. Oncotarget 8, 20834-20841 (2017).

10. Itzykson, R. et al. Prognostic score including gene mutations in chronic myelomonocytic leukemia. J. Clin. Oncol. 31, 2428-2436 (2013).

11. Plo, I. et al. An activating mutation in the CSF3R gene induces a hereditary chronic neutrophilia. J. Exp. Med. 206, 1701-1707 (2009).

12. Patnaik, M. M. et al. Prognostic interaction between ASXL1 and TET2 mutations in chronic myelomonocytic leukemia. Blood Cancer J. 6, e385 (2016).

13. Palomo, L. et al. Molecular landscape and clonal architecture of adult myelodysplastic/myeloproliferative neoplasms. Blood 136, 1851-1862 (2020) 